\title{
Advances in the Development of Encapsulants for Mercuric Iodide X-Ray Detectors
}

\author{
J. S. IWANCZYK, MEMBER, IEEe, Y. J. WANG, J. G. BRADLEY, A. L. ALBEE, AND W. F. SCHNEPPLE
}

\begin{abstract}
Advances in the development of protective impermeable encapsulants with high transparency to ultra-low-energy $X$-rays for use on $\mathrm{HgI}_{2} \mathbf{X}$-ray detectors are reported. Various $X$-ray fluorescence spectra from coated detectors are presented. The $X$-ray absorption in the encapsulants has been analyzed using characteristic radiation from various elements. Results suggest that low-energy cutoffs for the detectors are not determined solely by the encapsulating coatings presently employed but are also influenced by the front electrode and surface effects, which can affect the local electric field or the surface recombination velocity. An energy resolution of $182 \mathrm{eV}$ (FWHM) has been achieved for $\mathrm{Ni} L$ lines at $850 \mathrm{eV}$. Improved detector sensitivity to $\mathrm{X}$-ray energies under $700 \mathrm{eV}$ is demonstrated.
\end{abstract}

\section{INTRODUCTION}

$\mathbf{M}_{\mathrm{a}}^{\mathrm{E}}$ ERCURIC iodide has received considerable interest as a material useful for solid-state $\mathrm{X}$-ray detector spectrometer applications [1]-[3]. Bare $\mathrm{HgI}_{2}$ detectors are capable of operation to quite low photon energies, while retaining excellent resolution; however, proper $\mathrm{HgI}_{2}$ surface passivation and device encapsulation are critical for insuring their long term reliability. For the last several years we have made a major effort to develop a protective impermeable $\mathrm{X}$-ray transparent coating that will not degrade the detector performance, while still assuring good protection in various adverse environmental conditions. Several excellent protective surface treatments have been identified and previously reported, including polymethylmethacrylate (PMMA) and Parylene ${ }^{\circledR}[4]-[6]$.

The encapsulation of detectors introduces an additional $X$-ray attenuation by the protective material itself. This is particularly critical for low-energy $\mathrm{X}$-rays. In this paper we report on the results of recent efforts to improve the low-energy response of these detectors through the use of higher transparency coatings, while still maintaining, or even improving, long-term device reliability.

Results using Parylene-C as an encapsulant have shown that this material quite adequately meets the protection needs

Manuscript received January 17, 1990; revised June 26, 1990. This work was sponsored by the National Aeronautics and Space Administration and supported in part by NIH grant \#5R01 GM 37161.

J. S. Iwanczyk and Y. J. Wang are with Xsirius, Inc. (formerly the Institute of Physics, University of Southern California), 4640 Admiralty Way, Suite 214, Marina del Ray, CA 90292.

J. G. Bradley is with the Jet Propulsion Laboratory, California Institute of Technology, 4800 Oak Grove Drive, Pasadena, CA 91125.

A. L. Albee is with the California Institute of Technology, 1201 East California Blvd., Pasadena, CA 91125

W. F. Schnepple is with Xsirius Scientific, Inc., 4640 Admiralty Way, Suite 214, Marina del Ray, CA 90292.

IEEE Log Number 9038740.

${ }^{*}$ Registered service mark of Union Carbide Corporation. for $\mathrm{HgI}_{2} \mathrm{X}$-ray detectors [2]. In the interest of reducing the attenuation of the coating for very-low-energy X-rays, however, it appeared that some improvement could be gained by changing to Parylene-N. Unlike Parylene- $\mathrm{C}$ the Parylene-N composition contains no chlorine and should therefore be more transparent to low-energy $\mathrm{X}$-rays. To investigate this alternative coating, we initiated a program to adapt our Parylene-C processes and procedures to permit the deposition of thin films of Parylene- $\mathrm{N}$ and to perform testing on coated $\mathrm{X}$-ray detectors.

While the attenuation of the encapsulant has, until recently, been the most critical parameter in determining the low-energy cutoff of the response of the X-ray detectors, it is not the only factor. As a result of this present work, other important influences were found to be the attenuation caused by the detector's entrance electrode and also the condition of the detector surface, which can affect the electric field and the surface recombination velocity. These topics will be discussed in the paper.

\section{EXPERIMENTAL}

\section{A. Parylene Encapsulation}

We have found it impossible in the past to achieve good low-energy cutoffs with commercially supplied relatively thick Parylene-C coatings. The best of these coatings did not transmit X-rays below approximately $1000 \mathrm{eV}$ or sometimes even higher energies. As a consequence, we have devoted considerable program effort to developing and improving our own in-house Parylene-C deposition capability at the University of Southern California (USC) [2], [3]. The improved low-energy cutoffs obtained from our Parylene-C depositions have been accomplished primarily through a carefully controlled reduction of the thickness of the Parylene-C detector encapsulating material. This reduction in thickness was made possible because of the development of improved in-house deposition techniques, including carefully controlling the amount of Parylene material loaded into the source boat and its heat-up temperature ramp rate. As a result of the improved processing, the films which were deposited were highly transparent and completely free of the quantity of gases that are often present in commercially produced films, and which give them a cloudy or milky appearance. "Gas bubbles," if they occur in the films, increase the probability of the occurrence of pinholes in the film. As a consequence, "gas bubble" type films require a greater deposition thickness to insure a seal for the detector. The new clear films can still provide vacuum integrity for the detector but need only be in much thinner deposits. 
To enhance our control over the encapsulation process, we have developed a dynamic measurement technique that continuously monitors the thickness of the Parylene film during its deposition. Using an optical method frequently employed for the measurement of thin films, the technique detects the successive interference maxima and minima that are successively created as the Parylene thickness slowly builds up on a reflective witness slide placed near the detectors. A small $\mathrm{He}-\mathrm{Ne}$ laser provides a monochromatic light source for illuminating the witness slide. The intensity of the reflected beam is detected by a silicon photodiode whose amplified output is recorded on a strip chart recorder. Counting the number of successive maxima and minima recorded yields, through a simple calculation, an indication of the film's thickness. The method yields an accuracy of one-quarter wavelength or better for these coatings. In film thickness, this is equivalent to an uncertainty of less than $0.1 \mu \mathrm{m}$.

Although Parylene-C provides good protection, it does contain chlorine, a relatively high- $Z$ element. The chlorine provides for improved cross-linking of the polymer; however, it also produces undesirable strong attenuation for very soft X-rays. We therefore have made some experiments to deposit thin films of chlorine-free Parylene- $\mathrm{N}$, which should have less attenuation than the Parylene- $\mathrm{C}$ films of equal thickness. However, compared to Parylene-C deposition runs, these have proved to be much more difficult.

Initially, the resulting Parylene-N films were not clear but instead were cloudy in their appearance. In depositing Parylene, the evaporated dimer is first passed through a high-temperature region of the system, where it is cracked to the monomer. The Parylene- $\mathrm{N}$ monomer then passes to a plasma region, where cross-linking is enhanced and the polymer then deposits onto the detector surfaces [7]. The plasma was specially introduced into the Parylene- $\mathrm{N}$ processing procedures to create a film with improved physical properties. To reduce the cloudiness of the deposits, it was found necessary to raise the temperature of the hot zone considerably above that used for Parylene-C (i.e., $775^{\circ} \mathrm{C}$ versus $650^{\circ} \mathrm{C}$ ). In addition, there was a frequent tendency to produce powdery deposits on the detector. These appear to come from streaming of the uncracked dimer and unpolymerized monomer depositing directly onto the detector. Such deposits are of extremely poor quality in that the powder will subsequently come off, leaving a large number of pinholes in the film that remains. Further improvements in repeatability for this processing are needed; however, initial data from Parylene-N coated detectors are encouraging. An alternative deposition geometry, in which the detector surface to be coated is shielded by a baffle from the directly streaming particles, has already resulted in a much-improved deposit, free of pinholes. As described in the next section on detector results, the low-energy cutoff is improved with Parylene- $\mathrm{N}$ compared to that for Parylene-C.

\section{B. X-Ray Detector Testing and Results}

Windowless Detector Probe System: Low-energy X-ray testing of the detectors was done using both the scanning electron microscope and particle analyzer (SEMPA) instru- ment at the Jet Propulsion Laboratory (JPL) and a windowless detector probe test system assembled at USC. The SEMPA is a multifunction prototype instrument designed for SEM and XRF applications on NASA space missions. In this application, the $\mathrm{HgI}_{2}$ device is used as an XRF radiation detector to provide elemental analysis of the sample under test and must be stored and operated for extended periods of time in the high vacuum environmental conditions, which will exist on long duration space missions. Because $\mathrm{HgI}_{2}$ has a measurable vapor pressure even at room temperature, a gas-tight encapsulation coating is thus mandatory to prevent gradual sublimation loss of the material; however, at the same time, the coating should provide good transmission for $\mathrm{X}$-rays down to the characteristic energy of sodium (ca 1 $\mathrm{keV}$ ).

The important influences of both the Parylene coating and the near-surface electric field persuaded us of the need to have available at USC a quick and convenient method of evaluating the effects of changes in detector processing. Consequently, an XRF test system was built at USC using a modified Tracor analysis instrument. In this case X-rays rather than the electrons employed in the SEMPA were used as the primary radiation to excite various fluorescence targets. The system consisted of an X-ray generator tube (with rhodium anode and beryllium exit window), a rotating carousel containing multiple targets, and a probe head containing the $\mathrm{HgI}_{2}$ detector and its charge-sensitive preamplifier. The target carrier could be rotated without breaking vacuum to position any one of several targets into the primary X-ray beam from the tube. Fluorescence X-rays coming from the target were then detected by the probe head.

Because there was no window in front of the detector in the USC system, the detector probe, X-ray tube and sample holder were kept under continuous vacuum in order to prevent any moisture condensation during detector and FET cooling and to assure minimum absorption for low-energy $\mathrm{X}$-rays. The total volume evacuated was much larger than the volume inside the detector probe.

The USC system was used to excite radiation from a number of different targets, including $\mathrm{Al}, \mathrm{Cu}, \mathrm{Ni}$, a teflon specimen (for generating fluorine $\mathrm{K} \mathrm{X}$-rays, $677 \mathrm{eV}$ ). The $\mathrm{X}$-ray tube had a thin beryllium exit window and was operated at voltages up to $13 \mathrm{kV}$. This produced an output of not only the usual Bremsstrahlung spectrum but also fairly strong $L$ line radiations from the tube's rhodium anode.

The USC system had somewhat more electronic noise, due to certain construction specifics that were different on the USC probe, as compared to the noise obtained on the JPL SEMPA (175 eV versus $152 \mathrm{eV}$ FWHM pulser widths, respectively). This noise, however, was easily low enough to allow for evaluations and comparisons to be made between different detectors and coatings.

Fig. 1 presents a spectrum taken from the $\mathrm{HgI}_{2}$ detector probe (so-called Mark II version) showing a copper fluorescence spectrum as obtained on the JPL SEMPA instrument. The $\mathrm{Cu} L$ lines, at an energy of around $940 \mathrm{eV}$, are barely visible in this figure. The $\mathrm{HgI}_{2}$ detector used in the Mark II probe to take this spectrum was built using a Parylene-C 
Deteclor Test Results

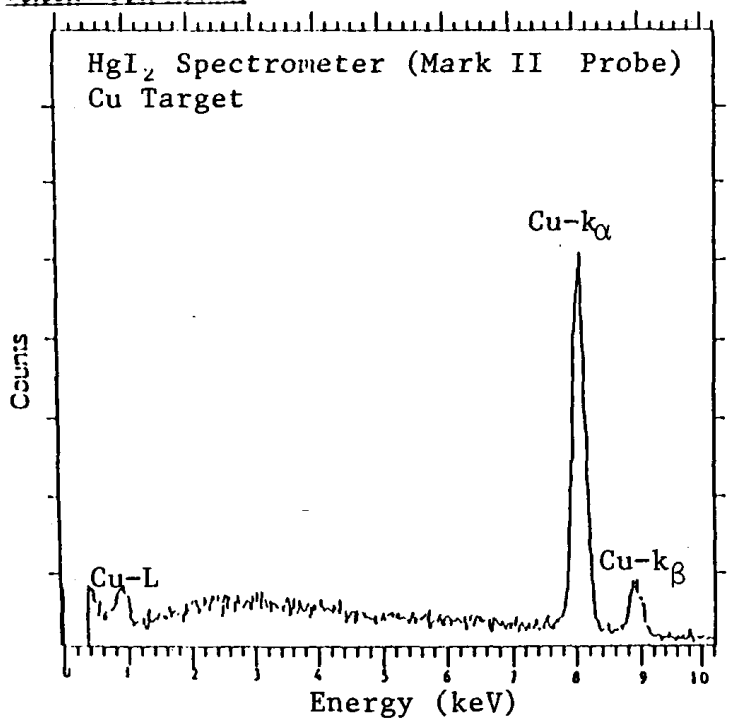

Fig. 1. X-ray fluorescence spectrum for copper.

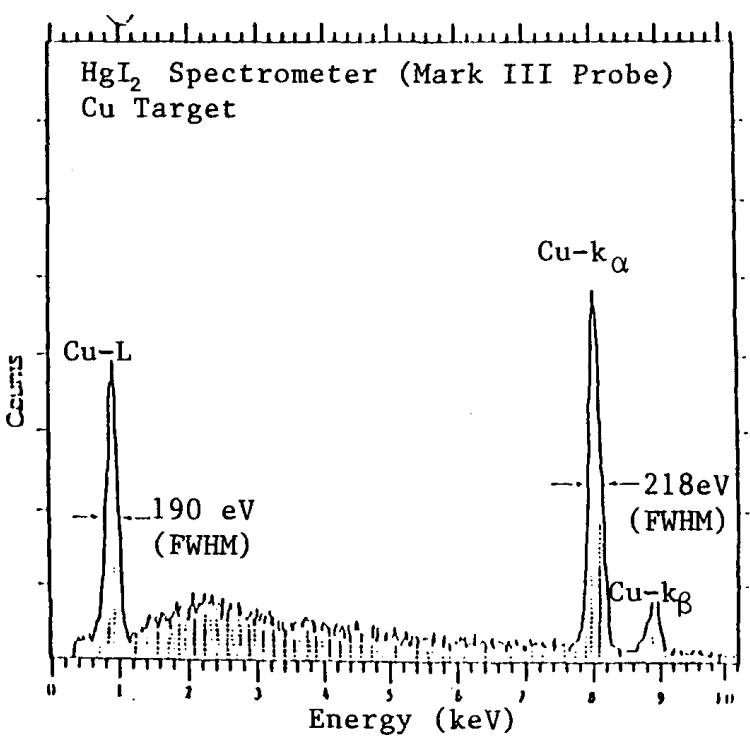

Fig. 2. X-ray fluorescence spectrum for copper.

coating supplied by an outside vendor. The encapsulation film thickness was estimated at 7-8 $\mu \mathrm{m}$, and its effect in reducing the sensitivity to the $L$ line is clearly apparent.

Fig. 2 shows another spectrum taken on the SEMPA using the same $\mathrm{Cu}$ fluorescence source; however, this time it is taken with a newer Mark III probe, which incorporated a detector encapsulated at USC. In contrast to Fig. 1, this spectrum clearly shows a higher-intensity well-defined (190 eV FWHM) peak for the combined $\mathrm{Cu} L$ lines $\left(L_{\alpha}, L_{\beta}\right.$ at $928,948 \mathrm{eV}$, respectively). The reduced attenuation from the much thinner (ca. $3.5 \mu \mathrm{m}$ ) USC Parylene-C coating is thus clearly evident. Also shown are the $K_{\alpha}$ and $K_{\beta}$ lines at 8.0

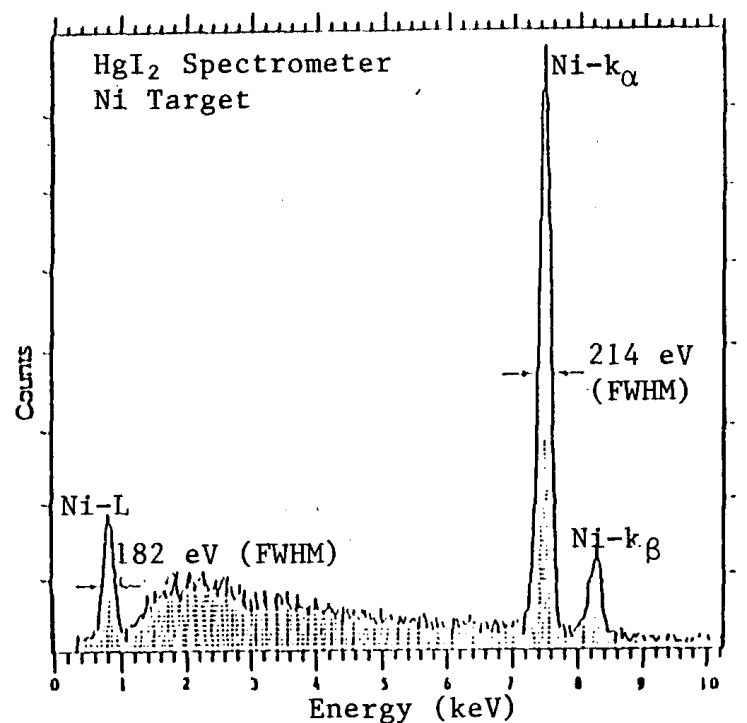

Fig. 3. X-ray fluorescence spectrum for nickel.

and $8.9 \mathrm{keV}$ (with resolution of $218 \mathrm{eV}$ for the $8.0 \mathrm{keV}$ peak).

Fig. 3 shows Mark III probe SEMPA instrument data taken with the same $\mathrm{HgI}_{2}$ detector as was used for Fig. 2 . The figure shows the fluorescence spectrum from a $\mathrm{Ni}$ target excited with electrons. In addition to the $\mathrm{Ni} K_{\alpha}$ and $K_{\beta}$ lines at 7.5 and $8.3 \mathrm{keV}$, the combined $\mathrm{Ni} L$ lines are clearly visible at $849 \mathrm{eV}$ with an energy resolution of $182 \mathrm{eV}$ (FWHM).

Fig. 4 shows four different spectra taken using the windowless USC system and a Parylene-C coated detector. The low-energy cutoff permits the transmission and detection at fluorine $K$-line energies, as shown by the well-defined peak at $677 \mathrm{eV}$. In addition to the fluorine $K_{\alpha}$ spectrum, three other fluorescent spectra peaks are shown in the figure: Ni $L$ $(0.86 \mathrm{keV}), \mathrm{Cu} L(0.94 \mathrm{keV})$, and $\mathrm{Al} K(1.486 \mathrm{keV})$. In all cases, the peaks are clearly resolved above the backgrounds.

A major objective of this testing was to study both the attenuation of the Parylene coatings and also changes in the detector front window transmission due to processing variations (e.g., in the Pd metallization). The approach employed was to measure the $\mathrm{X}$-ray responses of different detectors to both the $K$ and $L \mathrm{X}$-ray lines from elements such as $\mathrm{Cu}$ or $\mathrm{Ni}$. The concept was that the ratio of the $K / L$ responses for one detector could be compared to that for another detector, perhaps with a different coating, to assess low-energy sensitivity. $K / L$ ratios were used since under fixed excitation conditions, the ratios should be relatively independent of detector-to-detector geometry or configuration variations.

Based upon cross-section data for the thin Parylene coatings under consideration, photons at any of these $K$ line energies (ca. 7-8 keV) were all highly penetrating, i.e., the coatings were essentially transparent. The $L$ line photons on the other hand, were in the energy range $(<1 \mathrm{keV})$ where attenuation in the coating becomes very significant. Hence, any differences noted in the ratios for different detector/coat- 

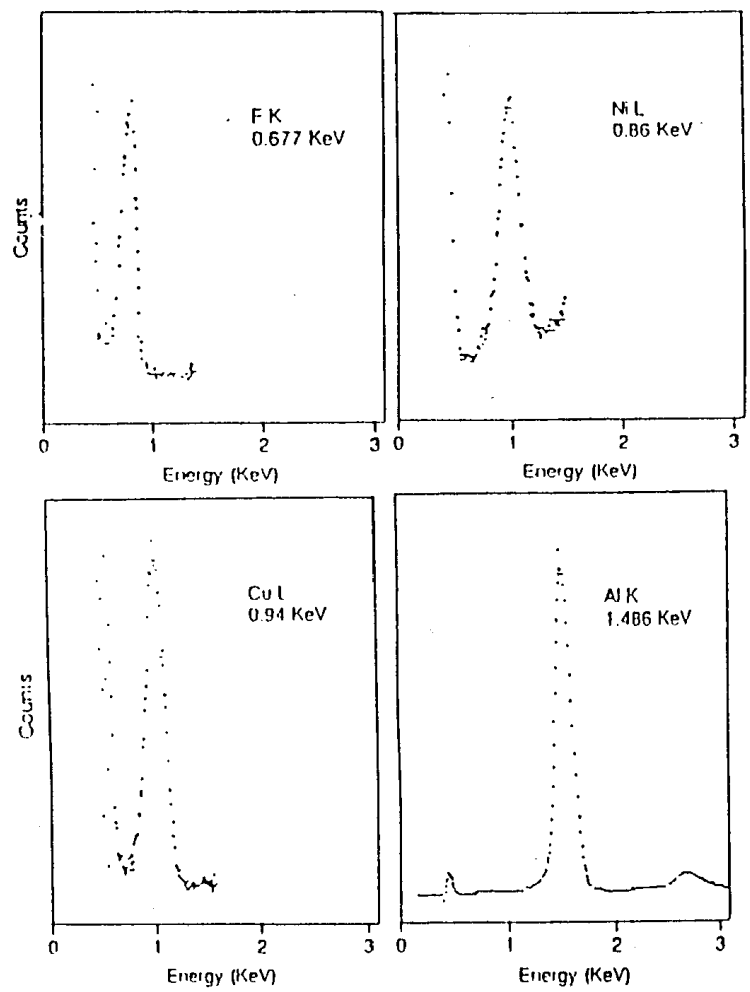

Fig. 4. X-ray fluorescence spectrum for $\mathrm{F}, \mathrm{Ni}, \mathrm{Cu}$, and $\mathrm{Al}$.

TABLE I

\begin{tabular}{|c|c|c|c|c|c|c|c|c|c|c|c|}
\hline \multirow{4}{*}{ Target } & \multirow{4}{*}{$\begin{array}{c}L \text { Line Energy } \\
\mathrm{eV}\end{array}$} & \multicolumn{7}{|c|}{ Calculated } & & & \\
\hline & & \multirow{2}{*}{$\begin{array}{c}\text { Parylene-N } \\
I / I_{0}, \%\end{array}$} & \multicolumn{3}{|c|}{$\begin{array}{c}\text { Parylene-C } \\
I / I_{0}, \%\end{array}$} & \multicolumn{3}{|c|}{$\begin{array}{c}\text { Ratio } \\
L_{P-N} / L_{P-C} \\
\text { Parylene-N }=3.6 \mu \mathrm{m} \\
\text { Parylene-C }=\end{array}$} & \multicolumn{3}{|c|}{ Experimental } \\
\hline & & & 3.3 & 3.5 & 3.7 & 3.3 & 3.5 & 3.7 & $P-N$ & $P-C$ & \\
\hline & & $\mu \mathrm{m}$ & $\mu \mathrm{m}$ & $\mu \mathrm{m}$ & $\mu \mathrm{m}$ & $\mu \mathrm{m}$ & $\mu \mathrm{m}$ & $\mu \mathrm{m}$ & $K_{\alpha} / L$ & $K_{\alpha} / L$ & $L_{P-N} / L_{P-C}$ \\
\hline $\mathrm{Cu}$ & 925 & 37.0 & 31.3 & 29.5 & 27.5 & 1.18 & 1.25 & 1.35 & 64 & 81 & 1.26 \\
\hline $\mathrm{Ni}$ & 849 & 28.6 & 23.5 & 21.5 & 20.0 & 1.22 & 1.33 & 1.43 & 209 & 292 & 1.40 \\
\hline
\end{tabular}

Note: $K_{\alpha} / L$ is the ratio of the transmitted $K_{\alpha}$ line and $L$ line X-rays intensities measured. $L_{P-N} / L_{P-C}$ is the ratio of the transmitted $L$ line $\mathrm{X}$-ray intensities through the Parylene- $\mathrm{N}$ and Parylene- $\mathrm{C}$ coatings.

ing combinations could be assigned, almost entirely, to differences in $L$ line responses.

Measuring the same detector at two different targets would also provide two $K / L$ ratios. Again, since the transparency of any of the coatings was very high for $K$ line energies, changes in the ratio responses could be attributed to different $L$ line responses. It was thought that these $K / L$ ratios for a unit could then be examined to see if they changed in agreement with an expected calculated change due to the known thickness of the Parylene coating. Determining absolute theoretical $K / L$ ratios existing at the front entrance window of the detector proved extremely difficult due to such things as geometry factors, the presence of a complicated excitation spectrum from the X-ray tube, self-absorption in the fluorescing target, etc. [8]. The ratio technique therefore was used only as a relative method to compare the low-energy sensitivity of one detector to another.

Table I shows calculated and measured data for two $\mathrm{HgI}_{2}$ detectors, one coated with Parylene- $\mathrm{N}$ and the other with Parylene-C. The theoretical X-ray percentage transmissions were calculated using linear attenuation coefficients calculated from cross sections appropriate to the two different compositions of the materials. The measured data were based upon a summation of the counts in a $K$ or $L$ peak in the output spectrum, which were above background. The thickness of $3.6 \mu \mathrm{m}$ cited for the Parylene-N coated detector was a value measured during deposition using the laser interference technique. The thickness of the Parylene- $\mathrm{C}$ coating on the other detector was not measured during its deposition run. Rather, a standard amount of dimer was processed, 
TABLE II

\begin{tabular}{|c|c|c|c|c|c|c|c|c|c|c|}
\hline \multirow{4}{*}{ Target } & \multirow{4}{*}{$\begin{array}{c}L \text { Line Energy } \\
\mathrm{eV}\end{array}$} & \multicolumn{9}{|c|}{ Calculated } \\
\hline & & \multicolumn{3}{|c|}{$\begin{array}{c}\mathrm{Pd} \\
I / I_{0}, \% \\
\end{array}$} & \multicolumn{6}{|c|}{$\underset{I / I_{0}, \%}{\mathrm{HgI}_{2}}$} \\
\hline & & 100 & 150 & 200 & 100 & 200 & 300 & 1000 & 4000 & 10000 \\
\hline & & $A$ & $\mathcal{A}$ & $A$ & $A$ & $A$ & $A$ & $A$ & $A$ & $A$ \\
\hline $\mathrm{Cu}$ & 925 & 92.5 & 86.7 & 82.0 & 94.6 & 89.4 & 84.5 & 57.1 & 10.6 & 0.37 \\
\hline $\mathrm{Ni}$ & 849 & 89.5 & 84.5 & 80.0 & 94.1 & 88.5 & 83.3 & 54.3 & 8.7 & 0.22 \\
\hline
\end{tabular}

which ordinarily gives a final film thickness on the detector of about $3.5 \mu \mathrm{m} \pm 0.2 \mu \mathrm{m}$. Calculated percentage transmission values for Parylene-C thickness of $3.3,3.5$, and $3.7 \mu \mathrm{m}$ are shown in the table.

Table II shows similar transmission values as calculated for various thickness of the Pd electrode and for a (possible) $\mathrm{HgI}_{2}$ dead layer. A Pd thickness of $150 \mathrm{~A}$ is the nominal value assumed for most of our metallization deposition runs. The thickness of an $\mathrm{HgI}_{2}$ dead layer is unknown; however, it is unlikely to be more than a hundred angstroms or so, since otherwise the low-energy sensitivities already achieved would not otherwise be possible.

\section{ANALysis of Results}

Reviewing the data in these tables, it appears that the experimental $L_{P-N} / L_{P-C}$ data in the last column of Table I matches closest the middle column data for the calculated $L_{P-N} / L_{P_{-C}}$ ratio. This corresponds to thickness of $3.5 \mu \mathrm{m}$ for the Parylene-C (calculated), and $3.6 \mu \mathrm{m}$ for the Parylene$\mathrm{N}$ (measured). While the agreement between the $\mathrm{Cu}$ ratio data is good ( 1.25 calculated versus 1.26 experimental), the lower-energy Ni data are less so. In this case, the calculated value of 1.33 is $5 \%$ lower than the experimental value of 1.40 .

While the true cause of the discrepancy is unknown at this time, several possibilities exist to explain the difference. First, something on the order of a $2 \%$ difference can be attributed simply to the increased attenuation for the lower energy $\mathrm{Ni} L$ line in the $\mathrm{Pd}$ front electrode, provided both the Parylene- $\mathrm{C}$ and Parylene- $\mathrm{N}$ detectors had the same Pd thickness. If there was some difference in the Pd thicknesses for the two detectors, as there might well have been, even more variation is possible (e.g., there is about $5 \%$ difference in transmission between 100 and $150 \mathrm{~A}$ films. Moreover, either a diminished electric field and/or a high surface recombination velocity near the front surface will reduce the charge signal collected in the detector and could also be invoked to help explain the discrepancy between the calculated and experimental ratios. These effects are conveniently described by an effective dead layer of $\mathrm{HgI}_{2}$ near the surface. The calculated attenuation for various assumed dead layer thicknesses are shown in Table II. The data are such that the existence of a dead layer cannot be precluded.

In therefore appears, on the basis of the data obtained, that any one or more of several different phenomena could be determining the low-energy sensitivity of these detectors. Further tests will be needed to clarify and resolve the relative importance of the separate elements of attenuation in the encapsulation coating, attenuation in the Pd electrode, and attenuation or electric field/surface recombination effects in the $\mathrm{HgI}_{2}$ itself.

\section{ConClusion}

Based upon the results obtained to date, we are optimistic about continued future progress in the areas of energy resolution, more optimal detector encapsulation, and enhanced sensitivity to low-energy X-rays. Examination of the $K / L$ line ratios appears to be a good technique for the relative ranking of different detectors based on their low-energy response. The data obtained so far, however, are insufficient to provide a clear-cut answer to the question of which factor, i.e., the coating, the near-surface electric field, or the recombination velocity, is presently limiting the low-energy cutoff. Future efforts will be directed towards gaining an improved understanding of these factors.

Even at the present state of development, however, $\mathrm{HgI}_{2}$ devices have already met the low-energy sensitivity and resolution needs for the JPL SEMPA instrument. These $\mathrm{HgI}_{2}$ detectors under development offer substantial advantages in terms of decreased power requirements, the absence of the need for cryogenic cooling, and fewer thermal/mechanical design difficulties. At the same time, their performance approaches that of cryogenically cooled $\mathrm{Si}(\mathrm{Li})$ units.

\section{ACKNOWLEDGMENT}

The valuable technical assistance of Mrs. F. Riquelme, Messrs. N. Dorri and B. Dancy of USC, and Mr. V. Taylor at JPL are greatly appreciated.

\section{REFERENCES}

[1] S. Swierkowki, G. Armantrout, and R. Wichner, "Recent advances with $\mathrm{HgI}_{2} \mathrm{X}$-ray detectors," IEEE Trans. Nucl. Sci., vol. NS-21, pp. $302-304,1974$.

[2] M. Slapa, G. Huth, W. Seibt, M. Schieber, and P. Randtke, "Capabilities of mercuric iodide as a room temperature X-ray detector," IEEE Trans. Nucl. Sci., vol. NS-23, no. 1, pp. 102-111, Feb. 1976.

[3] A. Dabrowski and G. Huth, "Toward the energy resolution limit of mercuric iodide in room temperature low energy X-ray spectrometry," IEEE Trans. Nucl. Sci., vol. NS-25, no. 1, pp. 205-211, Feb. IEEE

[4] J. S. Iwanczyk, "Advances in mercuric iodide X-ray detectors and low noise preamplification systems," Nucl. Instrumen. Methods, vol. A283, no. 2, pp. 208-214, Nov. 1989.

[5] J. S. Iwanczyk, Y. J. Wang, J. G. Bradley, J. M. Conley, A. L. Albee, and T. E. Economou, "Performance and durability of $\mathrm{HgI}_{2}$ $\mathrm{X}$-ray detectors for space missions," IEEE Trans. Nucl. Sci., vol. NS-36, no. 1, pp. 841-845, Feb. 1989.

[6] J. G. Bradley, J. M. Conley, A. L. Albee, J. S. Iwanczyk, A. J. Dabrowski, and W. K. Warburton, "Practical applications of $\mathrm{HgI}_{2}$ detectors to a space-flight scanning electron microscope," Nucl Instrumen. Methods, vol. A283, no. 2, pp. 348-351, Nov. 1989.

[7] J. M. Williams and J. T. Rowan, "Diagnosis and therapy for a recalcitrant Parylene coater," J. Vac. Sci. Technol., vol. A5, no. 4, Jul./Aug. 1987

[8] Eugene P. Bertin, Principles and Practice of X-ray Spectrometric Analysis. New York: Plenum, 1975, pp. 113-120. 